\title{
ON BLUETOOTH PHONEBOOK MANAGER
}

\author{
MOHANAD ABDULHAMID \\ AL-Hikma University, Iraq \\ E-mail: moh1hamid@yahoo.com
}

\begin{abstract}
The paper generally seeks to demonstrate an in depth understanding of computer communication using Bluetooth. The working principle of this technology has to be fully understood and thus the software required to save the phone book to the computer needs to be developed and implemented and thus demonstrated on how it works. The paper clearly illustrates how reliable the Bluetooth phone book manager is in terms of creating a backup of one's contacts. The software program is developed and then analysed in depth to understand the workability of the phone book manager which runs on any computer and is compatible with any phone. The hardware used is USB Bluetooth dongle, a cell phone and a computer.
\end{abstract}

Keywords: Bluetooth, phonebook manager, computer communication, ISM band, GUI.

\section{INTRODUCTION}

The problem requires one to develop an in depth understanding of the communication of devices using Bluetooth. The mode through which Bluetooth works has to be understood proficiently and special emphasis given to how the phone book can be saved onto the computer and then retrieved to the phone. It should be also compatible with any kind of cell phone $[1,2]$.

Issues of loss or theft of cell phones are on the rise in everyday life. Due to this, many people tend to lose their contacts which are usually saved on the phones and thus experience problems retrieving them. Thus, they tend to lose out on their network of people which they built over a period and getting back all the contacts becomes next to impossible. Therefore, the phone book manager takes a very integral step in solving this problem. The saving of the phone book through the Bluetooth to the computer will be very efficient as in case of loss of phone, the contacts of a person will still be available to him/her and then could be easily transferred to any other phone later on. The phone book will always be saved on the computer as a backup. It is also a very efficient and fast mode of communication as it is wireless and connects to any Bluetooth device within a certain radius [3, 4].

There are other modes of communication between portable devices say cell phones and computers for example the use of data cables or infrared but this technologies have limitations as in you have to be in front of the computer to transfer any data whereas for Bluetooth technology you can be at a radius of about 10 meters and it is usually very fast to transfer data compared to these other technologies.
Bluetooth is most likely going to be the most used and efficient mode of communications among most devices as its key features are robustness, low cost and low power, topping it up is it maintains high levels of security. Thus, making it a very attractive alternative as a means of communication $[5,6]$.

\section{DESIGN PROCEDURE}

This section describes the process involved in the software design. It marks many different modules to achieve various steps. They are all integrated on what each block is supposed to attain.

\subsection{System requirements}

The paper requires development of software that would receive contacts from a phone and store it, then also send the contacts to any phone as required. The program should do the following points:

○ Search for Bluetooth devices;

- Link with the available device;

- Receive data from the available Bluetooth device;

- Save the received data;

- Send the saved data to another Bluetooth device.

The software developed would be accessed by the user through a Graphical User Interface (GUI) which would help the user to either send or receive data. This means that the architecture of the developed software can be divided into 3 layers:

$\S$ Graphical user interface - This is responsible for giving the user options of whether to receive or send the saved data;

$\S \quad$ Model - This will take the requests from the user and respond accordingly based on the logic of the program;

$\S$ Program logic - This contains the system implementing functions.

\subsection{Receiving of contacts from phone}

The overall process of receiving of contacts from phone is shown in flowchart of Figure 1. The process is explained in the subsequent sections. 


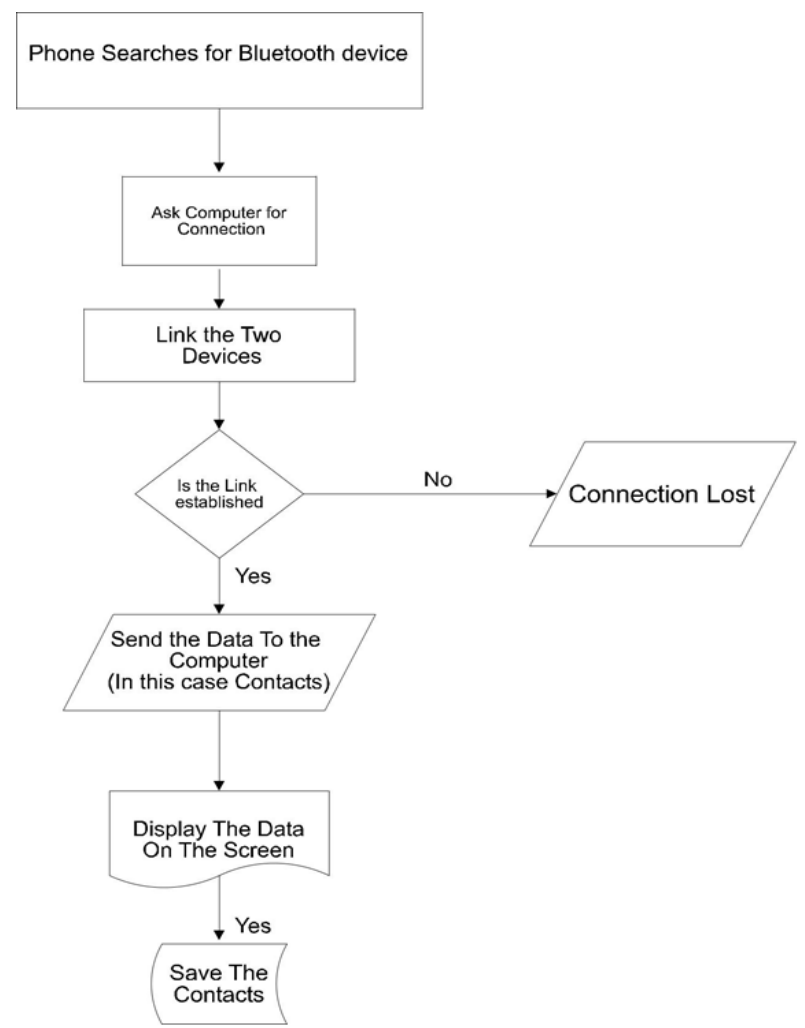

Figure 1. Receiving of contacts from phone

\subsubsection{Phone searches for Bluetooth device}

The phone's Bluetooth device searches for an active device i.e. the computer's device and displays the found device on its screen. The Bluetooth devices can search each other as they communicate at short-range radio links. They operate in the unlicensed industrial, scientific and medical (ISM) band at 2.4 Gigahertz hence are able to search for available Bluetooth devices.

\subsubsection{Ask computer for connection}

Once the search is completed and the available device has been listed on the phone screen, then it requests a connection with the computer. If the computer declines the request for connection from the phone, then we lose any further communication between the two devices but if it accepts the request then the link is established.

\subsubsection{Send data to computer}

After the request for a connection is granted, the phone again request the computer to accept the data which is being sent. If this request is rejected, then again we lose the connection between the devices. If the request is accepted then the phone starts transmitting the data (contacts) to the computer.

\subsubsection{Display data}

Once the data is received by the computer it will show what kind of data has been sent, in our case it should be vCard.

\subsubsection{Save the contact}

After the computer has displayed what kind of data is received then it is saved as a backup for future use.

\subsection{Sending of contacts to phone}

The overall process of sending of contacts to phone is shown in the flowchart of Figure 2. The process is explained in the subsequent sections.

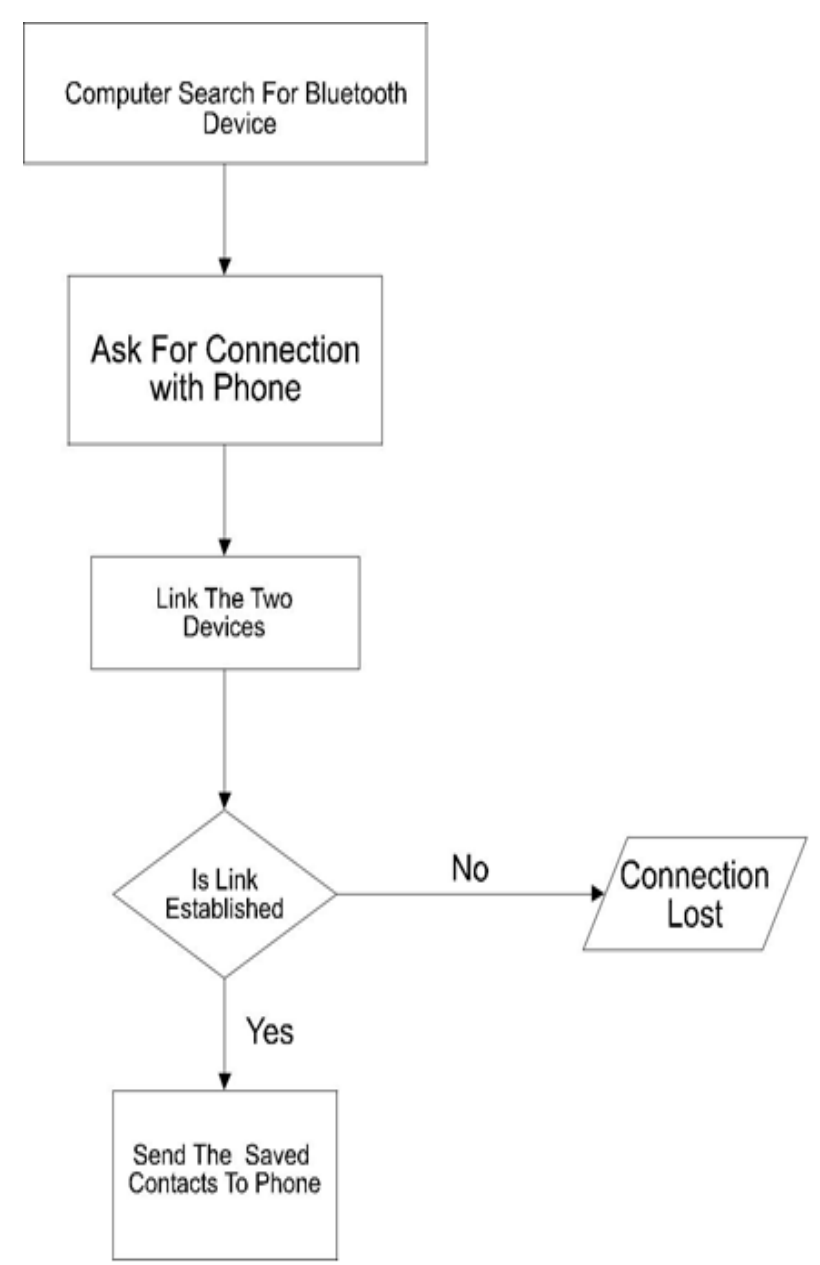

Figure 2. Sending of contacts to phone

\subsubsection{Computer searches for Bluetooth device}

The computer software will search for any active devices available. That is the phone with which we will be restoring the saved contacts. The available device will be listed on the screen.

\subsubsection{Ask for connection}

After the computer has searched for devices, it will request for a connection with the phone. If the phone device rejects the request for connection then the communication is lost, but if the device accepts the request then it moves to linking the devices. 


\subsubsection{Send contacts to phone}

After the connection has been established, the computer requests the phone to accept data that it is sending. If this request is declined then we lose the connection for sending data but if the request is accepted then the computer starts transmitting the contacts to the phone.

\section{RESULTS}

The software is developed in Java language. As shown in Figure 3, the software has its GUI which has a button "Discover Device" which is used to search for available active devices. The LocalDevice class method getDiscoverable is used to get the discoverable mode for a device.

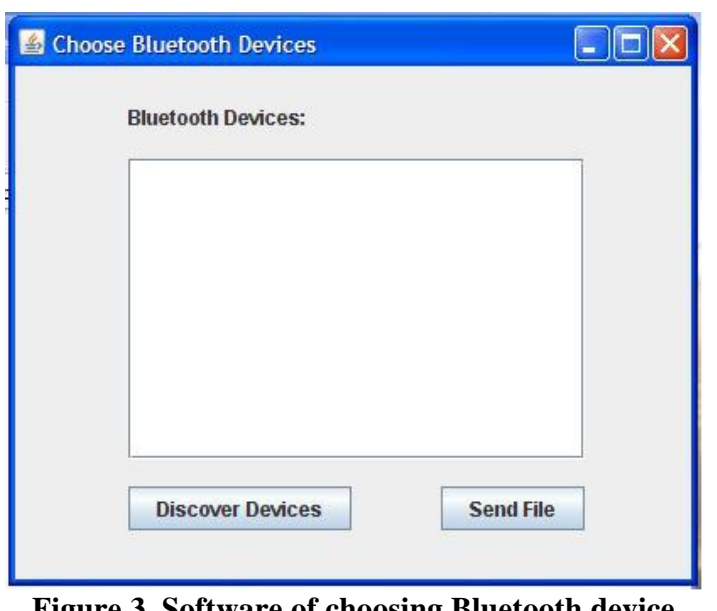

Figure 3. Software of choosing Bluetooth device

The DiscoveryAgent class provides the methods needed for performing device inquiry. Device inquiry is performed using the method startInquiry. Callbacks are made to an appropriate handler that implements the DiscoveryListener interface, via the methods deviceDiscovered (for each device found) and inquiryComplete (when the device inquiry process has ended).

Once the search is completed, it lists the device which is available for communication. After the device is displayed in the screen, we click on the second button which is "Send File". On clicking this button, a window pops up which gives the option of choosing which file to send to the linked device. Then we select the file that is to be sent and click on open. It starts to transmit the file to the linked device, it requests the other device to accept the file which is being sent. After it accepts to receive the file to the phone, the computer sends the file.

\section{CONCLUSION}

This paper discussed the requirements of developing a Bluetooth phone book manager. It is also mentioned the different formats in which the contacts are saved in a phone book of cell phones. The software developed was able to send saved contacts from the computer to the phone efficiently.

\section{REFERENCES}

[1] C. Bisdikian, "An overview of the Bluetooth wireless technology, " IEEE Communications Magazine, Vol.39, Issue 12, PP.86-94, 2001.

[2] Y. Liu, S. Yu, W. Chen, and W. Li, "Wireless communication technology based on Bluetooth and its application to a manipulator, " 4th IEEE International Conference on Industrial Informatics, Singapore, 2006.

[3] P. Singh, D. Sharma, and S. Agrawal, "A modern study of Bluetooth wireless technology, International Journal of Computer Science, Engineering and Information Technology, Vol.1, No.3, PP.55-63, 2011.

[4] A. Cotta, and N. Devidas, "Wireless communication using HC-05 Bluetooth module interfaced with Arduino," International Journal of Science, Engineering and Technology Research, Vol.5, Issue 4, PP.869-872, 2016.

[5] U. Rijah, and S. Mosharani, "Bluetooth security analysis and solution, " International Journal of Scientific and Research Publications, Vol.6, Issue 4, PP.333-338, 2016.

[6] B. Santhosh, K. Krithika, and K. Sunitha, "Research in communication mechanism between PC's Bluetooth and Android Bluetooth, " International Journal of Engineering Research and General Science, Vol.4, Issue 3, PP.424-431, 2016. 